\title{
Up-regulation of NK cell function against head and neck cancer in response to ss-isRNA requires TLR7
}

\author{
RALPH PRIES $^{1}$, SANDRA WULFF ${ }^{1}$, REBECCA KESSELRING ${ }^{1}$, \\ KIRSTEN BÖRNGEN ${ }^{1}$, LEI XIE ${ }^{2}$ and BARBARA WOLLENBERG ${ }^{1}$
}

\author{
${ }^{1}$ Department of Otorhinolaryngology, University of Schleswig-Holstein Campus Lübeck, D-23538 Lübeck, Germany; \\ ${ }^{2}$ Department of Head and Neck Surgery, Sir Run Run Shaw Hospital, Zhejiang University, 310016 Hangzhou, P.R. China
}

Received May 28, 2008; Accepted August 19, 2008

DOI: 10.3892/ijo_00000087

\begin{abstract}
Natural killer (NK) cells play a crucial role in innate immunity as effectors against tumor and pathogen-infected cells. NK-mediated host defense against tumor cells is strongly impaired in patients with head and neck squamous cell carcinoma (HNSCC). Tumor secretion of various immune suppressive mediators contributes to massively compromised immune functions. Herein we demonstrate that NK cell cytotoxicity against tumor cells of HNSCC can be efficiently stimulated by single-stranded immunostimulatory RNA (ss-isRNA). Stimulation with ss-isRNA results in an increased production of interferon- $\gamma$ and effector proteins perforin and granzyme B. Our investigations revealed that supernatants of permanent HNSCC cell lines negatively affect the ss-isRNAtriggered stimulation of cytolytic NK cell functions. Stimulation of cytotoxicity requires Toll-like receptor 7 (TLR7). Increased expression of NK cell TLR7 was shown in response to ss-isRNA. These results suggest ss-isRNA as a potential immunostimulatory tool of human NK cells against HNSCC.
\end{abstract}

\section{Introduction}

As effector members of innate immunity, natural killer (NK) cells play a major role in anti-infection activity and tumor surveillance. NK cells can directly kill target cells to which they are capable of adhering within 1-4 h without prior activation, priming or assistance by cytokines (1-3). NK cells can be triggered through various receptors depending on specific ligands presented by target cells in a given encounter (4-8). They are defined by the expression of CD56 and the presence of Fc $\gamma$ receptor III (CD16) (3). Two subsets of NK cells can be distinguished by the surface density of CD56,

Correspondence to: Dr Barbara Wollenberg, Department of Otorhinolaryngology, University of Schleswig-Holstein Campus Lübeck, Ratzeburger Allee 160, D-23538 Lübeck, Germany

E-mail: barbara.wollenberg@hno.uni-luebeck.de

Key words: natural killer cells, head and neck cancer, Toll-like receptor 7 namely the CD56 dim and bright subpopulations. The majority, approximately $90 \%$, of peripheral blood NK cells belong to the CD56 dim subset, which has been shown to be responsible for the natural cytotoxicity against tumor targets (9).

In contrast, NK cells isolated from cancer patients exhibit strongly impaired anti-tumor functions, which is also noted in head and neck squamous cell carcinoma (HNSCC) $(10,11)$. Cells of head and neck cancer are known to develop molecular strategies to escape efficient immune responses. It is believed that tumor production of various immune suppressive mediators contributes to massively compromised immune functions within the malignant transformation process (12-15).

It has been shown that treatment with the synthetic double-stranded (ds) RNA polyinosinic-polycytidylic acid (poly I:C), a mimic of a common product of viral infections, significantly up-regulates the cytotoxicity of human NK cells (16). Poly I:C is recognized by Toll-like receptor 3 (TLR3) (17), one of 10 different human TLRs which recognize conserved molecular patterns $(18,19)$. Our unpublished data demonstrate that TLR3 is also strongly expressed by cells of HNSCC where it participates in the regulation of tumor cell proliferation. These data stress the double-edged character of immunostimulatory approaches in anti-cancer therapies.

It has recently been reported that certain sequences of short interfering RNA (siRNA) are potent inducers of interferon- $\alpha$ $($ IFN $-\alpha$ ) in plasmacytoid dendritic cells (PDC), and therefore have been termed immunostimulatory RNA (isRNA). This additional biological activity of double-stranded (ds) isRNA was shown to be TLR7-dependent in human PDC, whereas it was demonstrated that the stimulation was not mediated by ssRNA molecules left over in the siRNA preparation after an incomplete annealing process (20). Previously, it was demonstrated that certain single-stranded (ss) RNA viruses are recognized by immune cells through TLR7, but specific sequences responsible for viral RNA recognition have not yet been identified (21-23). Based on the overlapping sequence of two siRNAs with potent IFN- $\alpha$-inducing activity, an immunostimulatory ssRNA sequence consisting of nine bases (5'-GUCCUUCAA-3') has been identified.

Our data indicate that single-stranded isRNA (ss-isRNA) is a potent inducer of NK cell function. Isolated human NK cells revealed a drastically increased cytotoxicity against 
cells of HNSCC in response to ss-isRNA stimulation. Analysis of cytokine secretion revealed that ss-isRNA as well triggers an increased production of interferon- $\gamma(\mathrm{IFN}-\gamma)$. In addition, extended expression levels of NK cell TLR7 were measured in response to ss-isRNA. The stimulatory activity of ss-isRNA requires functional TLR7 and is negatively affected by supernatants of permanent HNSCC cell lines.

\section{Materials and methods}

Isolation of NK cells. Human PBMC (peripheral blood mononuclear cells) were isolated from buffy coats provided by the blood bank of the University of Lübeck (Lübeck, Germany). Blood donors were 18- to 65-year-old healthy men and women who were tested and found negative for HIV, hepatitis B and hepatitis C viruses. PBMC were prepared from buffy coats by Ficoll-Hypaque density gradient centrifugation. Depletion of non-NK cells (T cells, B cells, dentritic cells, monocytes, granulocytes and erythroid cells) was used to isolate untouched NK cells with a cocktail of biotin-conjugated antibodies against CD3, CD4, CD14, CD15, CD19, CD36, CD123 and Glycophorin A, and Anti-Biotin MicroBeads, according to the manufacturer's protocol (Miltenyi Biotec, Bergisch Gladbach, Germany).

Cell culture. Permanent HNSCC cell lines BHY (DSMZ, Braunschweig, Germany) (24), PCI-1 and PCI-13 (hypopharyngeal cancer, Pittsburgh Cancer Institute, USA) were used to generate HNSCC supernatants. HNSCC cells as well as Daudi control cells were cultured in DMEM (Dulbecco's modified Eagle's medium, Gibco) supplemented with $10 \%$ FCS, $1 \mathrm{mM}$ glutamine and $0.1 \mathrm{mM}$ sodium pyruvate. Cell-free supernatants were collected by centrifugation and filtration after $48 \mathrm{~h}$ of cell cultivation. Isolated NK cells were incubated at $37^{\circ} \mathrm{C}$ in $5 \% \mathrm{CO}_{2}$ for $24 \mathrm{~h}$ in HNSCC supernatants or DMEM. For cell stimulation, isRNA was added to a final concentration of $100 \mathrm{nM}$ or $1 \mu \mathrm{M}$, respectively. Singlestranded immunostimulatory RNA (ss-isRNA) molecules (sequence, 5'-AGCUUAACCUGUCCUUCAA-3') were provided by Metabion, Germany, according to Hornung et al (20). The neutralizing anti-TLR7 antibody (IMG-581A) was purchased from Imgenex Corp. (San Diego, CA, USA).

Flow cytometry. Surface antigen staining was performed as described previously (25). Cells were stained with fluorescein isothiocyanate (FITC), phycoerythrin (PE), or adenomatous polyposis coli (APC)-conjugated antibodies (BD Biosciences). Propidium iodide was used to identify dead cells. For intracellular staining, NK cells were permeabilized with saponin buffer (PBS, $0.1 \%$ saponin, $1 \%$ FCS and $1 \mathrm{M}$ HEPES). Samples were analyzed on a FACSCanto (BD Biosciences), and data acquisition was performed using the FACS Diva software (BD Biosciences).

Cytolytic activity of NK cells. NK cells were analyzed for cytolytic activity using the CytoTox-96 Non-Radioactive Cytotoxicity Assay (Promega). This colorimetric assay quantitatively measures lactate dehydrogenase (LDH), a stable cytosolic enzyme that is released upon cell lysis, in much the same way as ${ }^{51} \mathrm{Cr}$ is released in radioactive assays. Released
LDH in culture supernatants was measured with a 30-min coupled enzymatic assay, which resulted in the conversion of a tetrazolium salt (INT) into a red formazan product as described previously $(26,27)$. The amount of color formed is proportional to the number of lysed cells. Visible wavelength absorbance data were collected using a standard 96-well plate reader. Several controls such as target cell spontaneous release and target cell maximum release, effector cell spontaneous LDH release, volume correction control, culture medium background, as well as the optimization of the target cell number were carried out according to the manufacturer's instructions. Data were expressed as the means \pm standard errors of the means (SEM). At least three independent experiments were conducted for each data point.

Cytokine analysis. Interferon- $\gamma$ was determined using the Bio-Plex Cytokine Assay (Bio-Rad) according to the instructions provided by the manufacturer. The Bio-Plex Cytokine Assay allows the analysis of multiple cytokines in a small volume of cell culture supernatant over a broad dynamic range (up to $0-32,000 \mathrm{pg} / \mathrm{ml}$ ). The principle of this assay is similar to a capture sandwich immunoassay but using spectrally addressed polystyrene beads coated with the corresponding antibodies. The cytokine assay was analyzed by a specialized microplate reader system (Bio-Plex Array Reader, Bio-Rad), and the revealed data were calculated using the Bio-Plex Manager software. Data were expressed as the means \pm SEM. At least three independent experiments were conducted for each data point.

Fluorescence microscopy. Fluorescence microscopy was used to analyze NK cell cytotoxicity against cells of HNSCC. Living cells were viewed on a Zeiss Axiovert 200M microscope by either differential interference contrast (DIC) microscopy or fluorescence microscopy using a green fluorescent protein (GFP) filter set (AHF Analysentechnik AG, Tübingen, Germany). Cells were photographed using a Zeiss AxioCam mrm rev.3 Firewire (D) and the Zeiss AxioVision Rel. 4.5 software. Cells of HNSCC were transfected with a GFP-conjugated nonsense oligonucleotide (Invitrogen) to achieve nonspecific cytoplasmic fluorescence which allowed the analyses of the cell lysis.

\section{Results}

Up-regulation of NK cell cytotoxicity against HNSCC in response to ss-isRNA. Human natural killer (NK) cells were isolated from peripheral blood and characterized by flow cytometry using phycoerythrin (PE)-labeled anti-CD56 antibodies. Our data revealed average purities of isolated NK cells of $\sim 95 \%$ (Fig. 1).

We evaluated whether single-stranded immunostimulatory RNA (ss-isRNA) had any effect on NK cell cytotoxicity against tumor target cells of HNSCC. Therefore, human NK cells were freshly isolated from peripheral blood and stimulated for $12 \mathrm{~h}$ with $100 \mathrm{nM}$ and $1 \mu \mathrm{M}$ ss-isRNA, respectively. As a control, NK cells were cultured in standard medium without ss-isRNA and in HNSCC supernatants. After $12 \mathrm{~h}$ of culture, NK cells were analyzed for their ability to lyse cells of permanent HNSCC cell lines. Spontaneous 


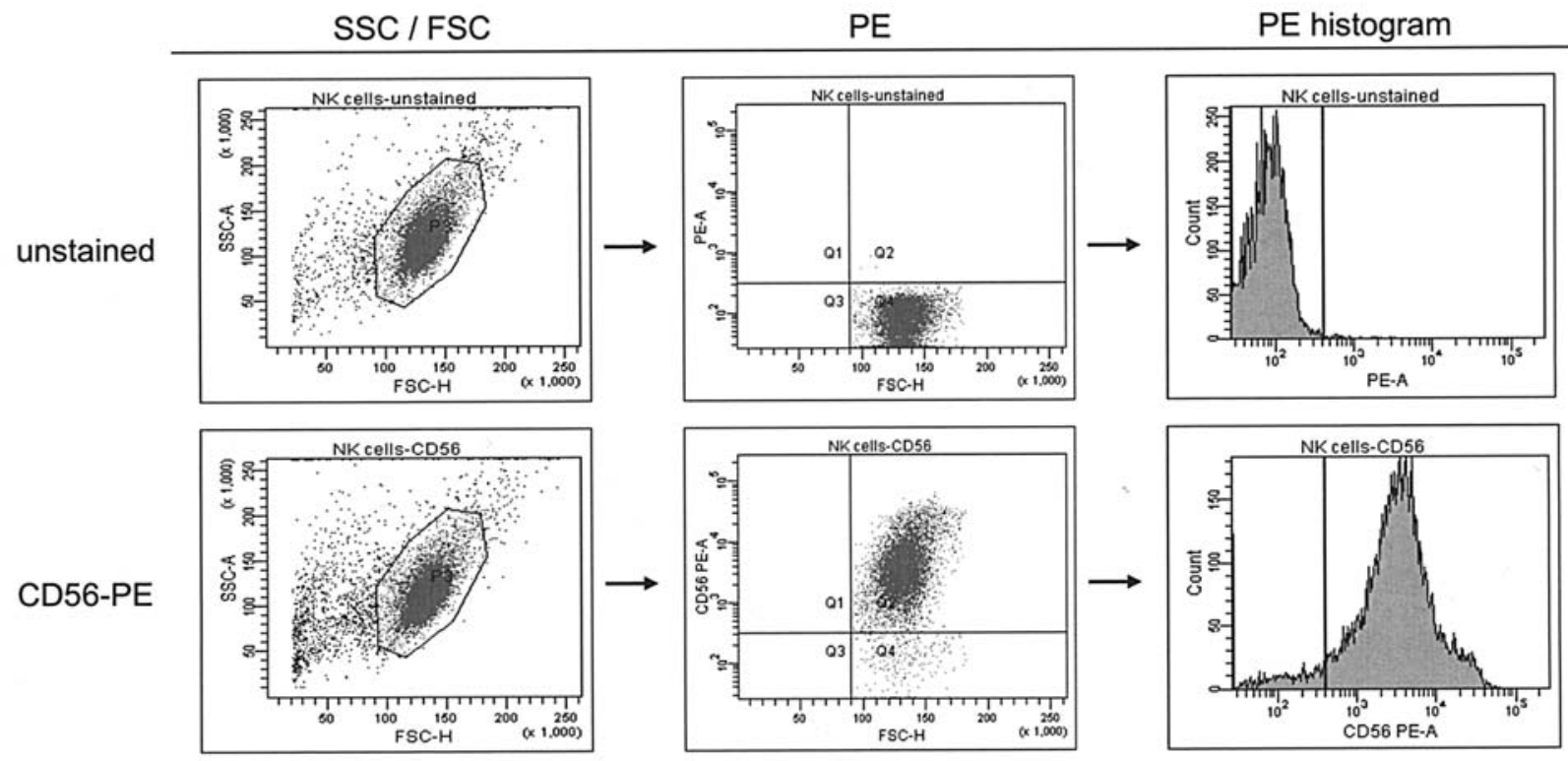

Figure 1. Flow cytometric analysis of isolated NK cells. Isolated NK cells were characterized by SSC/FSC properties and identified by the expression of the characteristic surface antigen CD56 using phycoerythrin (PE)-labeled anti-CD56 antibodies. NK cell isolation resulted in average purity of $\sim 95 \%$.

target cell lysis by unstimulated NK cells was approximately between 20 and $30 \%$, whereas NK cells efficiently killed between 80 and $95 \%$ of HNSCC target cells in response to stimulation with $1 \mu \mathrm{M}$ ss-isRNA (Fig. 2A). Cell lysis was also investigated using standard Daudi control cells which behaved in much the same way as cells of HNSCC. Stimulation with $100 \mathrm{nM}$ ss-isRNA resulted in a lower stimulation of cell lysis up to $65 \%$. NK cell culture in HNSCC supernatants resulted in a decreased NK cell cytotoxicity compared to the medium control (Fig. 2A).

Fluorescence microscopy of living cells was carried out to underline and visualize the demonstrated NK cell cytotoxicity against target cells of HNSCC in response to ss-isRNA stimulation. Therefore, cells of HNSCC were transfected with a GFP-conjugated nonsense oligonucleotide to achieve unspecific cytoplasmic fluorescence which allows the analyses of cell lysis. Transfected cells were incubated together with ss-isRNA-stimulated NK cells and NK cell cytotoxicity against HNSCC target cells was clearly illustrated by fluorescence microscopy (Fig. 2B).

To investigate the influence of the HNSCC microenvironment on ss-isRNA-stimulated cytotoxicity of NK cells, cells were simultaneously cultured with ss-isRNA and HNSCC supernatant as well as with ss-isRNA or HNSCC supernatant following a preincubation with HNSCC supernatant or ss-isRNA, respectively.

Our data revealed that HNSCC negatively affects the stimulation of NK cytotoxicity by ss-isRNA. However, ssisRNA-mediated stimulation of NK cells in the presence of HNSCC as well as before and after the incubation with HNSCC supernatant, still resulted in significantly increased levels of NK cytotoxicity compared to the medium control (Fig. 2C).

Induction of INF- $\gamma$ production of NK cells after stimulation by ss-isRNA. One of the primary effector functions of NK cells is the production of IFN- $\gamma$. As an additional parameter of NK cell function, the secretion levels of IFN- $\gamma$ were determined for ss-isRNA and HNSCC, respectively. NK cells were stimulated with $1 \mu \mathrm{M}$ ss-isRNA for $24 \mathrm{~h}$ in the presence and absence of HNSCC supernatant. Significantly increased levels of IFN- $\gamma$ secretion were measured in response to ssisRNA stimulation compared to the medium control. NK cell stimulation in the presence of HNSCC supernatant resulted in a lower increase in IFN- $\gamma$ secretion (Fig. 3A). These data underline the NK cell activating capacity of ss-isRNA as well as the inhibitory influence of HNSCC on this process.

Induction of granzyme $B$ and perforin production of NK cells after stimulation by ss-isRNA. One mechanism used by NK cells to kill tumor and virus-infected cells is the release of granzyme and perforin proteins from cytotoxic granules. Following adhesion of the cytotoxic cell to the target, granzyme B is directly exocytosed and enters the target cell, assisted by perforin. Granzyme B and perforin proteins induce apoptosis in target cells by forming transmembrane pores and through cleavage of effector caspases such as caspase-3. Since granzymes and perforin constitute the primary effectors of the granule exocytose pathway in NK cells, we analyzed the expression of these cytolytic mediators in response to ssisRNA stimulation using flow cytometry. Therefore, NK cells were stimulated as described above, and the expression of granzyme B and perforin was measured after $30 \mathrm{~min}$ of incubation with HNSCC target cells. Our data indicated a significant increase in both proteins compared to the medium controls (Fig. 3B).

In addition, CD56 bright and CD56 dim subpopulations of NK cells were analyzed in the presence and absence of isRNA. Although these two NK cell subpopulations are often distributed into 'regulatory' and 'cytotoxic' NK cells, the precise role of surface protein CD56 for NK cell function is still mostly unclear. Our data demonstrated that isRNA 
A

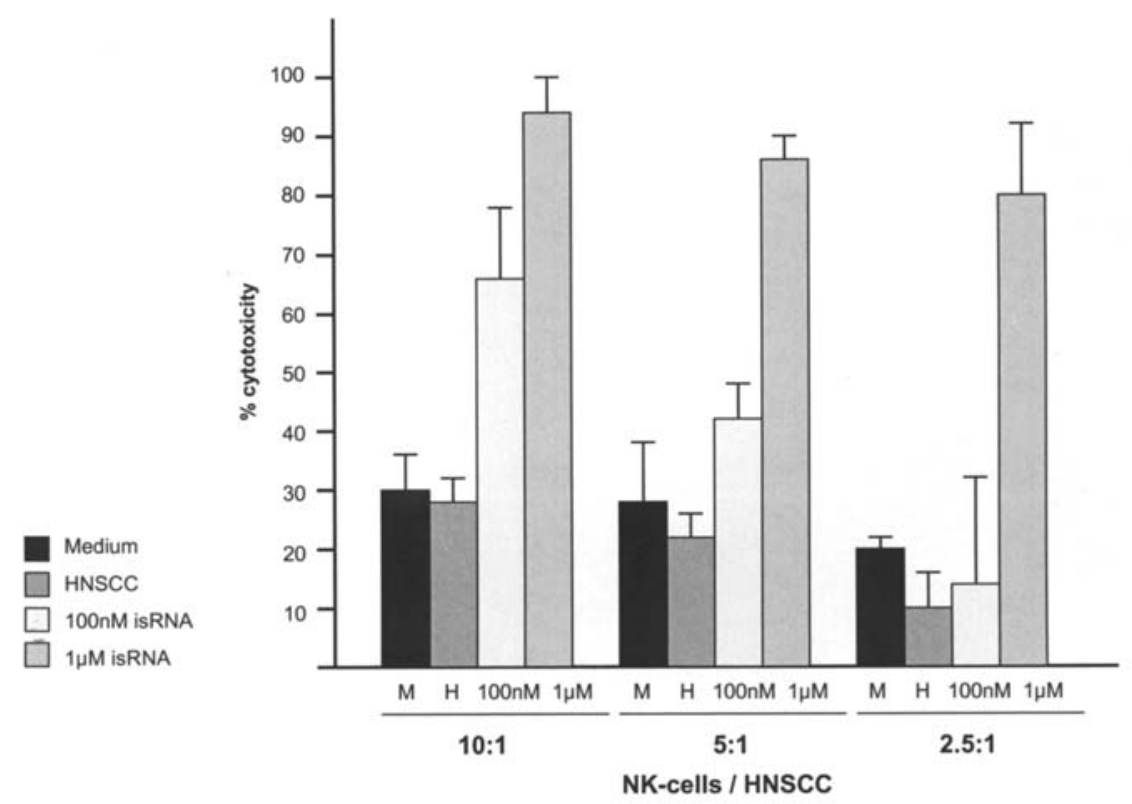

B

\section{5 hours of incubation}

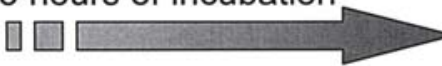

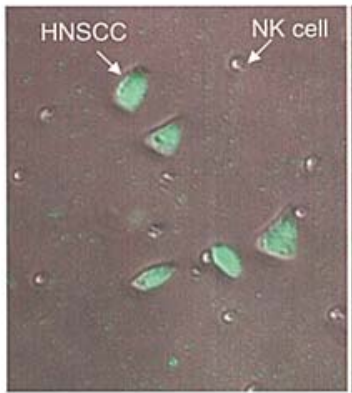

start of incubation

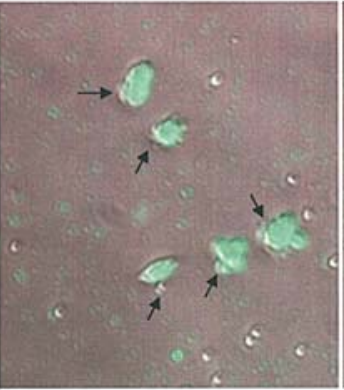

NK cell annealing

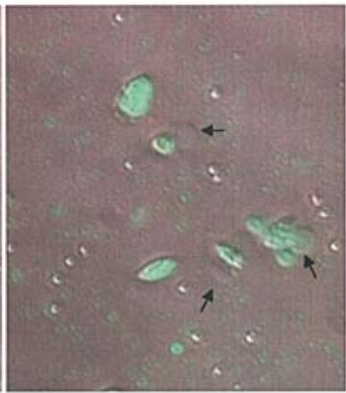

effusion of cytoplasm

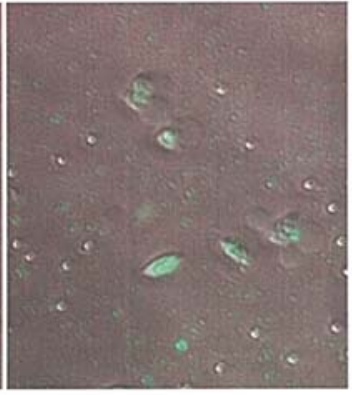

complete cell lysis

C

Medium

$12 \mathrm{~h} \mathrm{HNSCC} / 12 \mathrm{~h} 1 \mu \mathrm{M}$ isRNA

$24 \mathrm{~h} 1 \mu \mathrm{M}$ isRNA + HNSCC

$12 \mathrm{~h} 1 \mu \mathrm{M}$ isRNA / $12 \mathrm{~h} \mathrm{HNSCC}$

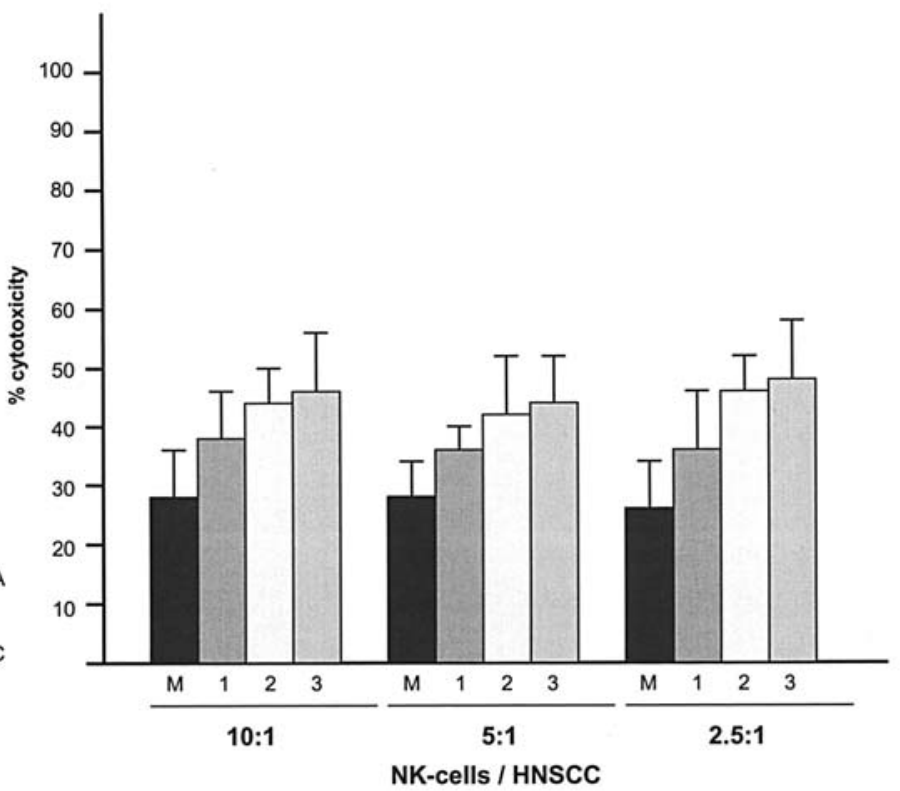

Figure 2. NK cell cytotoxicity against cells of HNSCC. (A) Human NK cells were freshly isolated from peripheral blood and stimulated for $12 \mathrm{~h}$ with $100 \mathrm{nM}$ and $1 \mu \mathrm{M}$ ss-isRNA, respectively. As a control, NK cells were cultured in standard medium without ss-isRNA and in HNSCC supernatants. After $12 \mathrm{~h}$ of culture, NK cells were analyzed for their ability to lyse cells of permanent HNSCC cell lines. Data are shown as the means \pm SEM. (B) Analyses of NK cell cytotoxicity by fluorescence microscopy of living cells. Cells of HNSCC were transfected with a GFP-conjugated nonsense oligonucleotide to achieve unspecific cytoplasmic fluorescence which allows the analyses of cell lysis. Transfected cells were incubated together with ss-isRNA-stimulated NK cells and analyzed for $6 \mathrm{~h}$. Annealing of NK cell and cell lysis of HNSCC target cells was clearly illustrated by fluorescence microscopy. (C) To investigate the influence of the HNSCC microenvironment on ss-isRNA-stimulated cytotoxicity of NK cells, cells were simultaneously cultured with ss-isRNA and HNSCC supernatant as well as cultured with ss-isRNA or HNSCC supernatant following a preincubation with HNSCC supernatant or ss-isRNA, respectively. Data are shown as the means \pm SEM. 
A

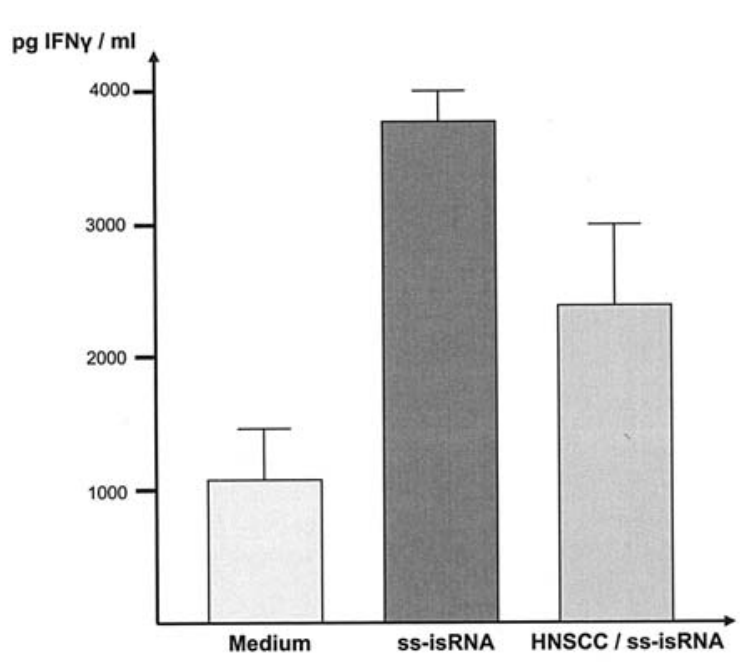

B
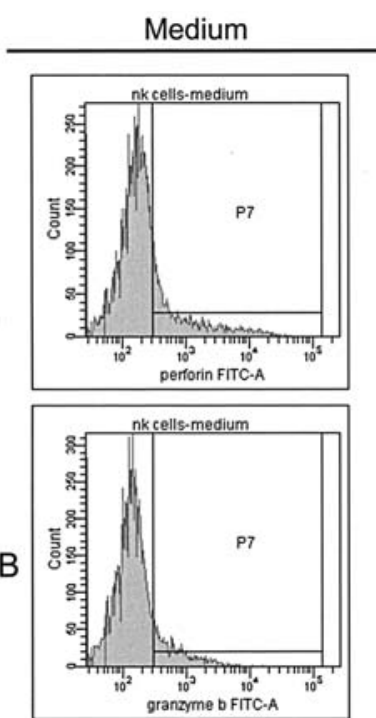

ss-isRNA
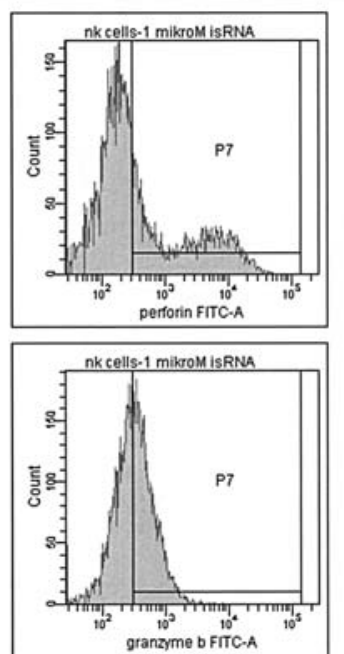

Figure 3. Production of cytokine interferon- $\gamma(\operatorname{IFN}-\gamma)($ A) and effector proteins perforin and granzyme B (B) in response to ss-isRNA and HNSCC. NK cells were stimulated with $1 \mu \mathrm{M}$ ss-isRNA for $24 \mathrm{~h}$ in the presence and absence of HNSCC supernatant. Medium incubated cells were used as a control. Data are shown as the means \pm SEM.

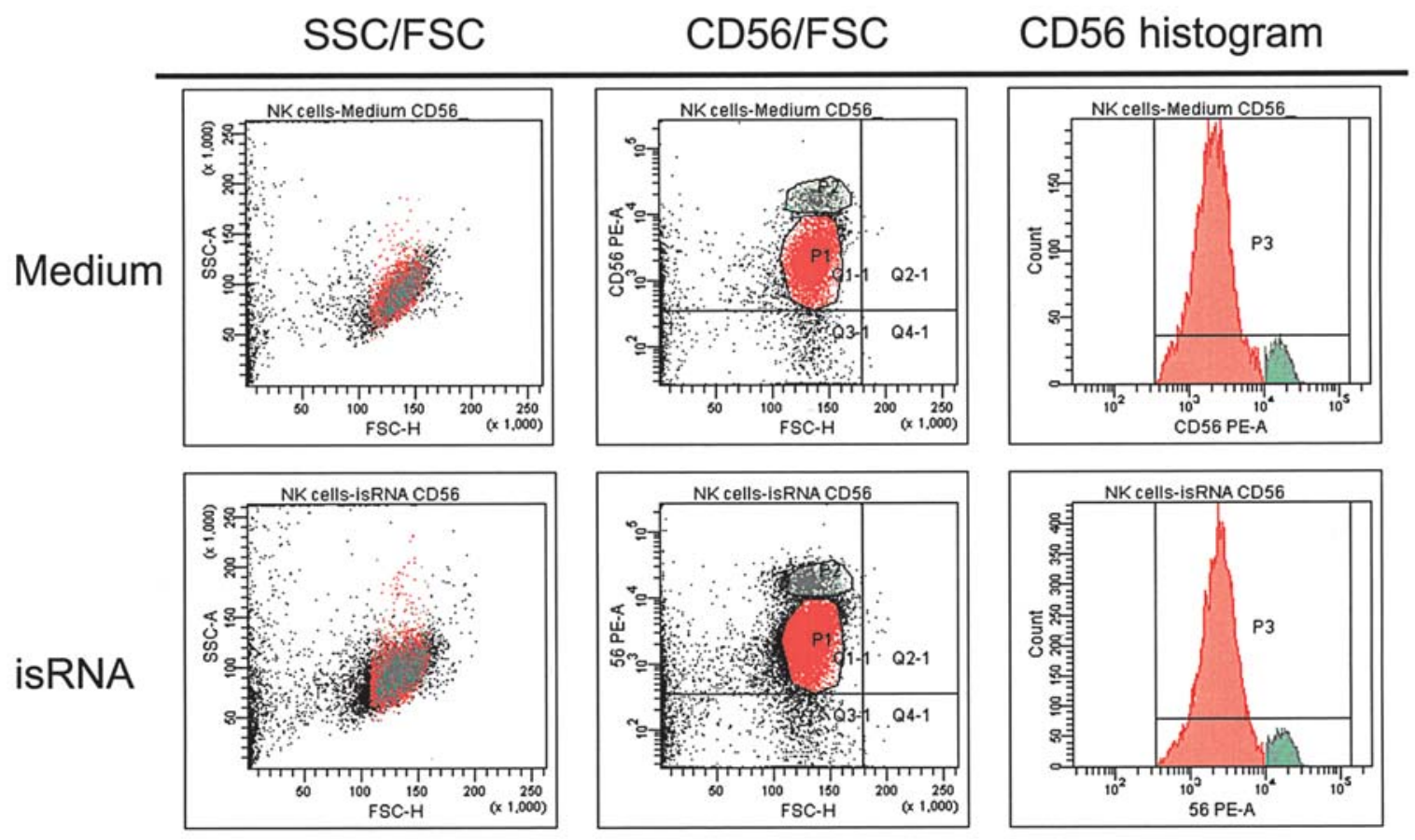

Figure 4. Influence of isRNA on NK cell subpopulations. CD56 bright and CD56 dim subpopulations of NK cells were analyzed in the presence and absence of $1 \mu \mathrm{M}$ isRNA. An average of $8 \%$ of the CD56 bright NK cells was found under both conditions.

stimulation had no significant effect on CD56 expression levels in these NK cell subsets (Fig. 4). Our data revealed an average of $\sim 8 \%$ of the CD56 bright NK cells in the presence as well as absence of isRNA. This suggests that there is no activation of regulatory NK cells in response to isRNA.

Increased TLR7 expression in response to ss-isRNA. Since TLR7 has been shown to be responsible for the isRNAmediated stimulation of plasmacytoid dendritic cells (20), we investigated the expression and regulation of TLR7 in human
NK cells in response to ss-isRNA and HNSCC. Therefore, cells were stimulated with ss-isRNA in the presence and absence of HNSCC as described above and then subjected to flow cytometric analyses of surface as well as intracellular expression levels of TLR7. As shown in Fig. 4, NK cell stimulation with $1 \mu \mathrm{M}$ ss-isRNA for $24 \mathrm{~h}$ led to an overall increase in TLR7 expression levels. Expression of TLR7 was significantly up-regulated on the cell surface as well as in the cellular lumen in response to ss-isRNA. The observed upregulation of TLR7 was slightly reduced in the presence of 


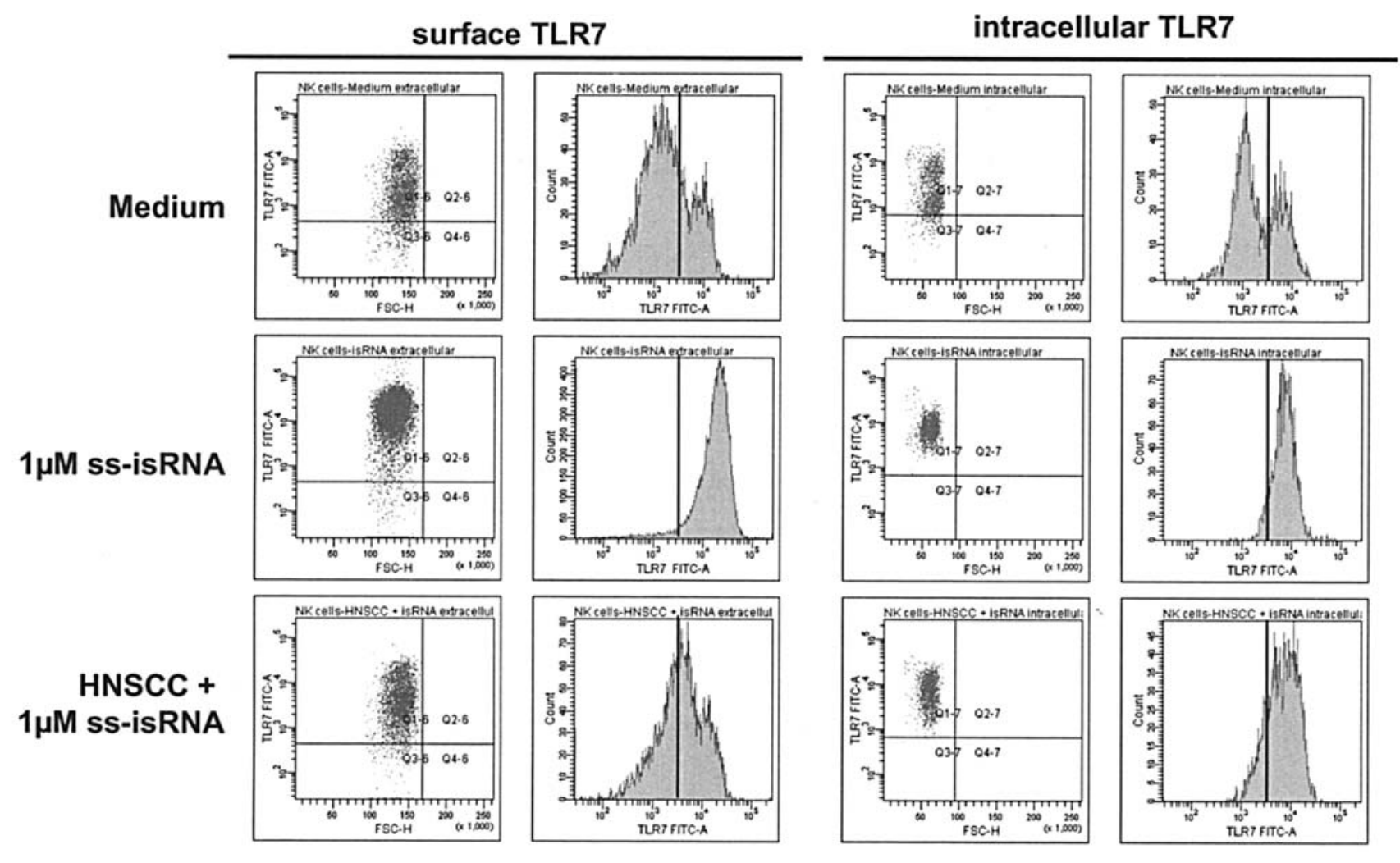

Figure 5. Expression of TLR7 in human NK cells. TLR7 expression was analyzed by flow cytometry in response to ss-isRNA and HNSCC. Cells were stimulated with ss-isRNA in the presence and absence of HNSCC as described above and then subjected to flow cytometric analyses of surface as well as intracellular expression levels of TLR7. NK cell stimulation with $1 \mu \mathrm{M}$ ss-isRNA for $24 \mathrm{~h}$ led to an overall increase in TLR7 expression levels on the cell surface as well as in the cellular lumen in response to ss-isRNA.

HNSCC supernatant (Fig. 5). These data suggest the direct involvement of TLR7 in the recognition and signal transduction of ss-isRNA.

Stimulation of NK cell cytotoxicity requires TLR7. To confirm the requirement of TLR7 for the ss-isRNA-mediated stimulation of human NK cells, we investigated whether an inhibition of TLR7 using TLR7-neutralizing antibodies would impair the stimulatory effect.

Therefore, isolated NK cells were preincubated with neutralizing anti-TLR7 antibodies and then stimulated with ss-isRNA as described. NK cell cytotoxicity was analyzed revealing that ss-isRNA-triggered stimulation was completely impaired by neutralizing anti-TLR7 antibodies. Mediumincubated NK cells as well as ss-isRNA-stimulated NK cells in the absence of anti-TLR7 antibodies were used as controls (Fig. 6). Surprisingly, NK cell cytotoxicity in response to TLR7 neutralization was found to be even lower than the medium control (Fig. 6). These data indicate that TLR7 is required for the recognition of ss-isRNA molecules and the subsequent stimulation of NK cell cytotoxicity and suggest furthermore that TLR7 might participate in additional regulatory routes or intercellular communication processes in human NK cells.

Our investigations showed no effects of ss-isRNA on different aspects of HNSCC tumorigenesis such as proliferation and cytokine production (data not shown) and suggest ss-isRNA as a potential immunostimulatory tool against HNSCC.

\section{Discussion}

NK cells express receptors that allow them to recognize pathogens and activate effector functions such as cytotoxicity and cytokine production (reviewed in ref. 28). Among these receptors are Toll-like receptors (TLRs) that recognize conserved pathogen structures and initiate innate immune responses (18). Since NK cell-mediated cytotoxicity represents a very important defense mechanism against tumor cells, the development of immunostimulating strategies of these cells is of great interest.

We demonstrated that cytotoxicity as well as IFN- $\gamma$ secretion of human NK cells can be triggered by singlestranded (ss) immunostimulatory (is) RNA molecules. Stimulation of human NK cells with ss-isRNA resulted in a strongly activated cytotoxicity against tumor cells of head and neck squamous cell carcinoma (HNSCC). The immunostimulatory potential of ss-isRNA was mediated through TLR7 and was affected by HNSCC (Fig. 7). The sequence of the isRNA used in these investigations was adapted to a sequence which was previously shown to possess a highly activating capacity on human plasmacytoid dendritic cells (20). It needs to be further elucidated whether isRNA sequence modifications may result in increased NK cell stimulation.

It has been demonstrated that NK cells can be activated by double-stranded RNA (dsRNA) via TLR3, whereas the molecular mechanisms are still mostly unknown $(18,29)$. Previous studies have demonstrated that human NK cells from peripheral blood express functional TLR3 and TLR9 

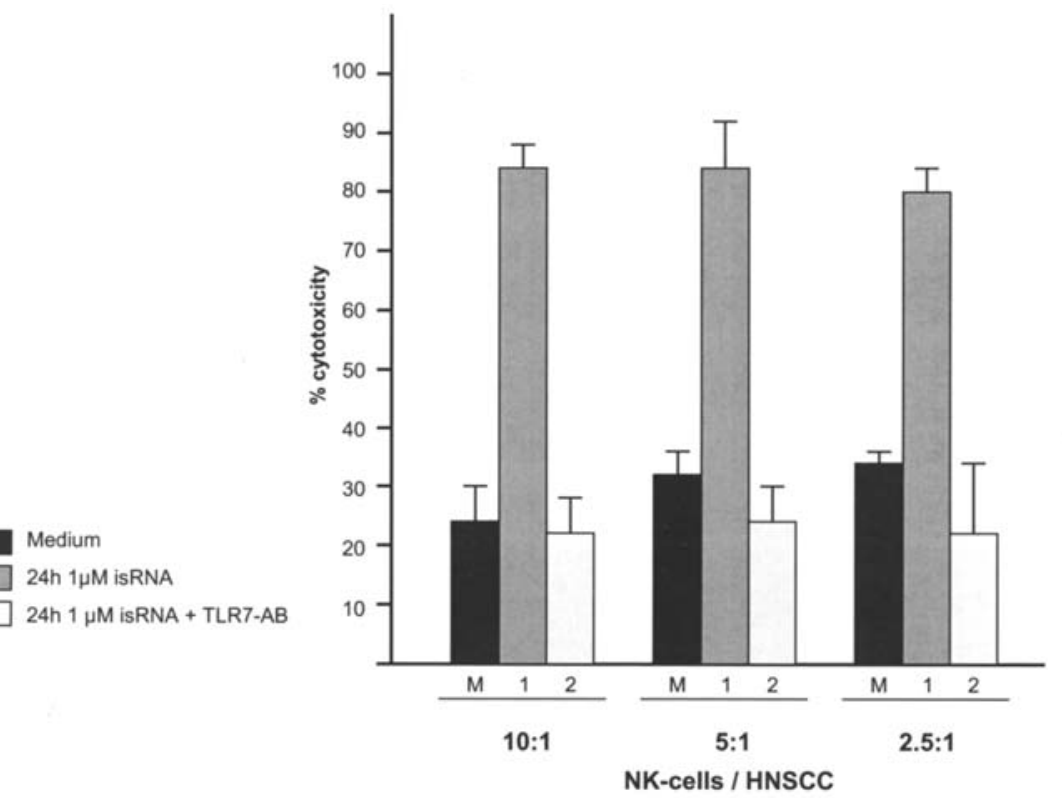

Figure 6. Stimulation of NK cell cytotoxicity requires TLR7. Isolated NK cells were preincubated with neutralizing anti-TLR7 antibodies and then stimulated with ss-isRNA as described. NK cell cytotoxicity was analyzed revealing that ss-isRNA-triggered stimulation was completely impaired by neutralizing antiTLR7 antibodies (TLR7-AB). Medium-incubated NK cells as well as ss-isRNA-stimulated NK cells in the absence of anti-TLR7 antibodies were used as controls. Data are shown as the means \pm SEM.

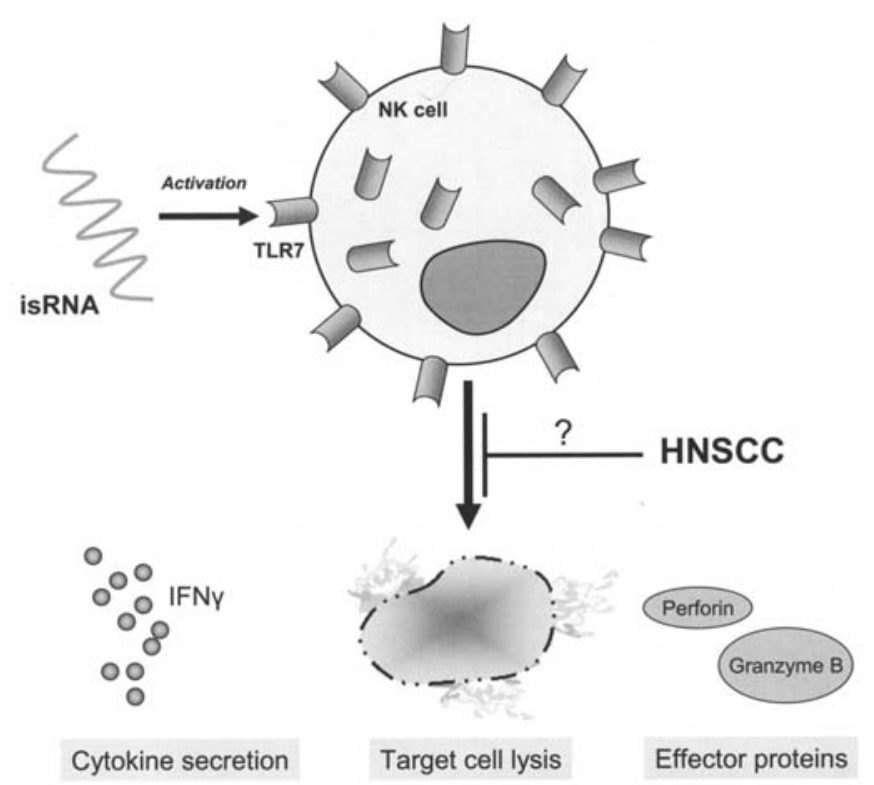

Figure 7. Model of NK cell stimulation through TLR7. Single-stranded isRNA molecules are recognized by TLR7 and lead to increased expression levels of cytokine IFN- $\gamma$ and effector proteins perforin and granzyme B. Activated NK cell functions result in increased target cell lysis. See text for details.

that allow responses to microbial products such as dsRNA (virus-derived) and $\mathrm{CpG}$ motifs (of bacterial origin). In response to these stimuli, NK cells became capable of producing IFN- $\gamma$ as well as tumor necrosis factor $\alpha$ (TNF- $\alpha)$ and acquired cytolytic activity against immature dendritic cells (iDCs) (30).
O'Connor and co-workers demonstrated that human NK cells also express TLR7 and TLR8 and that these receptors are functional (31). They showed that R848, an agonist of human TLR7 and TLR8, activates NK cell cytotoxicity against Daudi target cells. R848 was, as well, found to stimulate significant IFN- $\gamma$ production by NK cells (32).

Thus, it is becoming more and more evident that various receptors of the innate immune system allow NK cells to sense distinct pathogen-associated molecular patterns (PAMPS) and that the sum of these receptor systems determines the NK cell activation (31).

NK cell activity is known to be suppressed by a number of factors including surgery, cytotoxic drugs, stress, smoking or cytokines such as PGE2 (prostglandin E2) and IL-10, which also contribute to massively affected immune functions in head and neck cancer $(14,15)$. Novel approaches to antitumor therapies that augment natural resistance mediated by NK cells show much promise as an effective treatment in advanced malignant disease. Recently, lymphokine-activated killer (LAK) cells were transferred to patients with esophageal cancer just after surgery, when a postoperative immunosuppression cascade was proposed. It was found that immunosuppressive cytokines such as IL-6 and IL-10 significantly increased postoperatively, followed by a profound decrease in helper and cytotoxic T-cell subsets (33). These observations must be taken into account when developing novel immunostimulating strategies against different types of human cancer.

It has been suggested that neoplastic processes may modulate proper TLR signalling in various immune cells to advance cancer propagation $(34,35)$. In addition, it has been shown that Toll-like receptors are expressed by many cancer cell types and are potentially involved in tumor progression $(36,37)$. These data underline the doubled-edged character of TLR-stimulating strategies against human cancers. 


\section{Acknowledgements}

We are grateful to Ewelina Szymanski and Brigitte Wollmann for their skillful support in several sections of this work and all members of the Department of Otorhinolaryngology for the helpful discussions and a comfortable atmosphere. This work was supported by grants of the Werner and Klara Kreitz-Stiftung, the Monika-Kutzner-Stiftung, the PossehlStiftung, and the Rudolf-Bartling-Stiftung to B.W.

\section{References}

1. Trinchieri G: Biology of natural killer cells. Adv Immunol 47: 187-376, 1989.

2. Biron CA, Nguyen KB, Pien GC, Cousens LP and SalazarMather TP: Natural killer cells in antiviral defense: function and regulation by innate cytokines. Annu Rev Immunol 17: 189-220, 1999.

3. Cooper MA, Fehniger TA and Caligiuri MA: The biology of human natural killer-cell subsets. Trends Immunol 22: 633-640, 2001.

4. Moretta L, Bottino C, Cantoni C, Mingari MC and Moretta A: Human natural killer cell function and receptors. Curr Opin Pharmacol 1: 387-391, 2001.

5. Biassoni R, Cantoni C, Pende D, Sivori S, Parolini S, Vitale M, Bottino $\mathrm{C}$ and Moretta A: Human natural killer cell receptors and co-receptors. Immunol Rev 181: 203-214, 2001.

6. Moretta A, Bottino C, Vitale M, Pende D, Cantoni C, Mingari MC, Biassoni R and Moretta L: Activating receptors and coreceptors involved in human natural killer cell-mediated cytolysis. Annu Rev Immunol 19: 197-223, 2001.

7. Raulet DH, Vance RE and McMahon CW: Regulation of the natural killer cell receptor repertoire. Annu Rev Immunol 19: 291-330, 2001.

8. Lanier LL: Turning on natural killer cells. J Exp Med 191: 1259-1262, 2000.

9. Penack O, Gentilini C, Fischer L, Asemissen AM, Scheibenbogen C, Thiel E and Uharek L: CD56dimCD16neg cells are responsible for natural cytotoxicity against tumor targets. Leukemia 19: 835-840, 2005.

10. Bose A, Ghosh D, Pal S, Mukherjee KK, Biswas J and Baral R: Interferon alpha2b augments suppressed immune functions in tobacco-related head and neck squamous cell carcinoma patients by modulating cytokine signaling. Oral Oncol 42: 161-171, 2006.

11. Wang MB, Lichtenstein A and Mickel RA: Hierarchical immunosuppression of regional lymph nodes in patients with head and neck squamous cell carcinoma. Otolaryngol Head Neck Surg 105: 517-527, 1991.

12. Pries R, Thiel A, Brocks C and Wollenberg B: Secretion of tumor-promoting and immune suppressive cytokines by cell lines of head and neck squamous cell carcinoma. In Vivo 20: 45-48, 2006.

13. Pries R and Wollenberg B: Cytokines in head and neck cancer. Cytokine Growth Factor Rev 17: 141-146, 2006.

14. Douglas WG, Tracy E, Tan D, Yu J, Hicks WL Jr, Rigual NR, Loree TR, Wang Y and Baumann H: Development of head and neck squamous cell carcinoma is associated with altered cytokine responsiveness. Mol Cancer Res 2: 585-593, 2004.

15. Chin D, Boyle GM, Theile DR, Parsons PG and Coman WB: Molecular introduction to head and neck cancer (HNSCC) carcinogenesis. Br J Plast Surg 57: 595-602, 2004.

16. Pisegna S, Pirozzi G, Piccoli M, Frati L, Santoni A and Palmieri G: p38 MAPK activation controls the TLR3-mediated upregulation of cytotoxicity and cytokine production in human $\mathrm{NK}$ cells. Blood 104: 4157-4164, 2004.

17. Dunne A and O'Neill LA: The interleukin-1 receptor/Toll-like receptor superfamily: signal transduction during inflammation and host defense. Sci STKE 2003: re3, 2003.

18. Sivori S, Falco M, Della Chiesa M, Carlomagno S, Vitale M, Moretta L and Moretta A: CpG and double-stranded RNA trigger human NK cells by Toll-like receptors: induction of cytokine release and cytotoxicity against tumors and dendritic cells. Proc Natl Acad Sci USA 101: 10116-10121, 2004.
19. Sieling PA and Modlin RL: Toll-like receptors: mammalian 'taste receptors' for a smorgasbord of microbial invaders. Curr Opin Microbiol 5: 70-75, 2002.

20. Hornung V, Guenthner-Biller M, Bourquin C, Ablasser A, Schlee M, Uematsu S, Noronha A, Manoharan M, Akira S, de Fougerolles A, Endres S and Hartmann G: Sequence-specific potent induction of IFN-alpha by short interfering RNA in plasmacytoid dendritic cells through TLR7. Nat Med 11: 263-270, 2005.

21. Diebold SS, Kaisho T, Hemmi H, Akira S and Reis e Sousa C: Innate antiviral responses by means of TLR 7-mediated recognition of single-stranded RNA. Science 303: 1529-1531, 2004.

22. Heil F, Hemmi H, Hochrein H, Ampenberger F, Kirschning C, Akira S, Lipford G, Wagner $H$ and Bauer S: Species-specific recognition of single-stranded RNA via toll-like receptor 7 and 8. Science 303: 1526-1529, 2004.

23. Lund JM, Alexopoulou L, Sato A, Karow M, Adams NC, Gale NW, Iwasaki A and Flavell RA: Recognition of singlestranded RNA viruses by Toll-like receptor 7. Proc Natl Acad Sci USA 101: 5598-5603, 2004.

24. Kawamata H, Nakashiro K, Uchida D, Harada K, Yoshida H and Sato M: Possible contribution of active MMP2 to lymphnode metastasis and secreted cathepsin $\mathrm{L}$ to bone invasion of newly established human oral-squamous-cancer cell lines. Int $\mathbf{J}$ Cancer 70: 120-127, 1997.

25. Sozzani S, Luini W, Borsatti A, Polentarutti N, Zhou D, Piemonti L, D'Amico G, Power CA, Wells TN, Gobbi M, Allavena P and Mantovani A: Receptor expression and responsiveness of human dendritic cells to a defined set of CC and CXC chemokines. J Immunol 159: 1993-2000, 1997.

26. Korzeniewski C and Callewaert DM: An enzyme-release assay for natural cytotoxicity. J Immunol Methods 64: 313-320, 1983.

27. Decker T and Lohmann-Matthes ML: A quick and simple method for the quantitation of lactate dehydrogenase release in measurements of cellular cytotoxicity and tumor necrosis factor (TNF) activity. J Immunol Methods 115: 61-69, 1988.

28. Lopez-Botet M, Bellon T, Llano M, Navarro F, Garcia P and de Miguel M: Paired inhibitory and triggering NK cell receptors for HLA class I molecules. Hum Immunol 61: 7-17, 2000.

29. Schmidt KN, Leung B, Kwong M, Zarember KA, Satyal S, Navas TA, Wang F and Godowski PJ: APC-independent activation of NK cells by the Toll-like receptor 3 agonist double-stranded RNA. J Immunol 172: 138-143, 2004.

30. Marshall JD, Heeke DS, Abbate C, Yee P and Van Nest G: Induction of interferon-gamma from natural killer cells by immunostimulatory CpG DNA is mediated through plasmacytoid-dendritic-cell-produced interferon-alpha and tumour necrosis factor-alpha. Immunology 117: 38-46, 2006.

31. O'Connor GM, Hart OM and Gardiner CM: Putting the natural killer cell in its place. Immunology 117: 1-10, 2006.

32. Hart OM, Athie-Morales V, O'Connor GM and Gardiner CM: TLR7/8-mediated activation of human NK cells results in accessory cell-dependent IFN-gamma production. J Immunol 175: 1636-1642, 2005.

33. Yamaguchi Y, Hihara J, Hironaka K, Ohshita A, Okita R, Okawaki M, Matsuura K, Nagamine I, Ikeda T, Ohara M and Hamai Y: Postoperative immunosuppression cascade and immunotherapy using lymphokine-activated killer cells for patients with esophageal cancer: Possible application for compensatory anti-inflammatory response syndrome. Oncol Rep 15: 895-901, 2006.

34. Coussens LM and Werb Z: Inflammation and cancer. Nature 420: 860-867, 2002.

35. Killeen SD, Wang JH, Andrews EJ and Redmond HP: Exploitation of the Toll-like receptor system in cancer: a doubled-edged sword? Br J Cancer 95: 247-252, 2006.

36. Andrews EJ, Wang JH, Winter DC, Laug WE and Redmond HP: Tumor cell adhesion to endothelial cells is increased by endotoxin via an upregulation of beta-1 integrin expression. J Surg Res 97: 14-19, 2001.

37. Wang JH, Manning BJ, Wu QD, Blankson S, Bouchier-Hayes D and Redmond HP: Endotoxin/lipopolysaccharide activates NFkappa B and enhances tumor cell adhesion and invasion through a beta 1 integrin-dependent mechanism. J Immunol 170: 795-804, 2003. 